1. MBBS, FCPS

Post Graduate Trainee in Surgical Unit1 ,

Holy Family Hospital, Rawalpindi.

2. MBBS, FCPS (General surgery).

Senior Registrar

Dr Akbar Niazi Teaching Hospital IMDC, Islamabad.

3. MBBS

Student 4th Year

Rawalpindi Medical University, Rawalpindi.

4. MBBS, FCPS (General Surgery). Senior Registrar Surgical Unit-1, Holy Family Hospital, Rawalpindi.

5. MBBS, FCPS

Trainee Surgical Unit-1 Holy Family Hospital, Rawalpindi.

6. MBBS, FCPS (Anesthesia)

Assistant Professor Anesthesia Holy Family Hospital, Rawalpindi.

7. MBBS, FCPS

Trainee Surgical Unit-1

Holy Family Hospital, Rawalpindi.

8. MBBS, FCPS

Trainee Surgical Unit-1

Holy Family Hospital, Rawalpindi

9. MBBS, FCPS

Trainee Surgical Unit-1,

Holy Family Hospital, Rawalpindi.

10. MBBS, FCPS, FRCS, FACS

Professor Surgical Unit-1.

Holy Family Hospital.

Correspondence Address:

Dr. Ghulam Khadija

Department of Surgical Unit-1,

Holy Family Hospital, Rawalpindi

gkhadija63@gmail.com

Article received on:

28/12/2020

Accepted for publication:

23/02/2021

\section{Intravenous analgesia versus thoracic epidural catheterization in moderately severe pancreatitis: A randomized controlled trial in a tertiary care health facility.}

Ghulam Khadija ${ }^{1}$, Waqas Hasan Syed ${ }^{2}$, Sana Ullah ${ }^{3}$, Munazzah Aziz ${ }^{4}$, Sumaira Nawaz, Abeera Zareen ${ }^{6}$, Mehwish Changeez $^{7}$, Sara Malik ${ }^{8}$, Maham Tariq ${ }^{9}$, Jahangir Sarwar Khan ${ }^{10}$

ABSTRACT... Objective: To evaluate the two most commonly used methods for pain relief in acute pancreatitis i.e. epidural analgesia and I/V analgesia and compared the results. Study Design: Randomized Controlled Clinical Trial. Setting: Surgical Unit-1 of Holy Family Hospital, Rawalpindi. Period: June 2019 to June 2020. Material \& Methods: Patients presenting with acute pancreatitis with moderate severity were divided into groups A and B. In group A patients, epidural catheter was passed at T9-T10 level epidural space and they received $0.125 \%$ Bupivacaine injection every 4 hours, while group B patients received combination analgesia in the form of IV tramadol 100mg TDS and IV Toradol 30mg BD. Pain was assessed by using visual analog scale (VAS) at 12 hour intervals. Rescue analgesia, in the form of IV paracetamol $1 \mathrm{~g}$ given in the case of $>7$ VAS score was also recorded. Results: Total 100 patients were included according to the inclusion criteria of the study. Patients were randomly divided into two groups; Group A (Epidural) and group B (intravenous). Mean age (years) in the study was $42.39+11.21$ whereas there were 37 male and 63 female patients who were included in the study. In our study, mean pain score in group A was $3.16+1.23$ which was significantly lower than group $B(5.42+1.01), p$-value $<0.0001$. There was a single mortality in the study. 6 patient's required ventilatory support due to respiratory complications, 5 in group B and 1 in group A. Conclusion: Epidural analgesia is superior to I/V analgesics in pain management of moderately severe pancreatitis and it also reduces respiratory morbidity in these patients.

Keywords: $\quad$ Catheterization, Intravenous Analgesia, Pancreatitis, Paracetamol.

Article Citation: Khadija G, Syed WH, Sana Ullah, Aziz M, Nawaz S, Zareen A, Changeez M, Malik S, Tariq M, Khan JS. Intravenous analgesia versus thoracic epidural catheterization in moderately severe pancreatitis: A randomized controlled trial in a tertiary care health facility. Professional Med J 2021; 28(10):14011406. https://doi.org/10.29309/TPMJ/2021.28.10.6303

\section{INTRODUCTION}

Acute pancreatitis is an inflammatory condition of pancreas whose incidence in United States of America has reached 35/100000 population. ${ }^{1}$ In $80 \%$ of patients the disease is mild and self -limiting but it is the deadliest disease of hepatobiliary system in $20 \%$ of cases in which the mortality reaches up to $30 \%$ due to multiorgan failure and pancreatic necrosis. ${ }^{1}$ The etiology of acute pancreatitis varies among different areas and cultures but gallstones and alcohol consumption are major causes making 40-70\% of cases. Sudden onset of severe abdominal pain is hallmark of acute pancreatitis commonly localized to epigastric region and radiates like a belt around trunk into back. ${ }^{2}$ Severity of pain correlates with severity of acute pancreatitis and serum lipase and $\mathrm{C}$ reactive protein is diagnostic and prognostic indicator of acute pancreatitis respectively. ${ }^{3-5}$

Besides endoscopic removal of gall stones, treatment of acute pancreatitis is mainly supportive which includes fluid resuscitation, effective analgesia, early nutrition and oxygenation. ${ }^{2}$ These approaches do not affect pancreas directly but try to attenuate systemic inflammatory response and multiorgan failure.

Pain relief is mainstay of treatment. Analgesics used mostly for pain relief in acute pancreatitis are parenteral opioids and NSAIDS or their combination. 5,6 Epidural analgesia is now widely used to decrease pain and severity of disease in 
moderate to severe acute pancreatitis. ${ }^{1,3}$ Epidural analgesia blocks noxious afferent sympathetic nerve which improves pancreatic perfusion and decreases the severity of metabolic acidosis and tissue injury which prevent pancreatic necrosis. ${ }^{1,2,7}$

Although epidural analgesia is known to be far more effective than any IV analgesic, Epidural catheter insertion itself is an invasive procedure with known complications and thus many centers in the world are still reluctant to use it in pancreatitis. This study aims to provide evidence base for the use of epidural analgesia in acute pancreatitis and evaluates its efficacy in pain management of patients with moderately severe acute pancreatitis and compares it with IV analgesia.

\section{MATERIAL \& METHODS}

The study design was randomized controlled clinical trial carried out in surgical unit 1 of Holy Family Hospital, Rawalpindi for a period of 1 year i.e. from $16^{\text {th }}$ June 2019 to $15^{\text {th }}$ June 2020. Sample size was calculated using WHO sample size calculator, which came out to be 50 in each group. All the patients, male and female between 18 and 60 years with clinical features of acute pancreatitis, having serum amylase or lipase level greater than 3 times the upper normal limit with CTSI (CT scan severity index) between 4-8 (moderately severe), were included in our study. Patients having chronic pancreatitis, pancreatic cancer, hypersensitivity to bupivacaine, having any contraindication of epidural catheter placement (coagulation disorders, skin infection of vertebral site, spinal surgery etc.), breast feeding or pregnant and those who were referred from other centers after treatment were excluded from this study.

After approval from Institutional Ethics Research Forum Rawalpindi Medical College (RMC) (R-18$\mathrm{RMH}$ ), all the patients fulfilling the selection criteria reporting at surgical unit-I, Holy Family Hospital, Rawalpindi were informed about the study and written informed consent was taken.

A random number list was generated for 100 patients randomly allocating them in two groups group A or Group B (50patients in each) using SPSS software. CT scan abdomen with pancreatic protocol was performed in patients presenting with epigastric pain with raised amylase or lipase and CT severity index was assessed. Patients with CT severity index of 4-8 was included in the study. Standard treatment of acute pancreatitis which include IV fluids, antibiotics and PPI's remained same for all the patients.

Epidural catheter was passed by anesthetist in epidural space (T9-T10) in group A patients. These patients were given $0.125 \%$ bupivacaine via epidural route every 4-6 hourly and in group $B$ patients, pain relief was done by combination of intravenous opioids and NSAIDS i.e. injection tramadol 100mg IV TDS and injection Toradol $30 \mathrm{mg}$ IV BD. Pain was assessed on visual analog scale at 12-hour intervals. Rescue analgesia in the form of IV paracetamol $1 \mathrm{~g}$ was given if the VAS score exceeded 7 and requirement of rescue analgesia for each group was also recorded and analyzed. Data was analyzed using SPSS version 22.0.

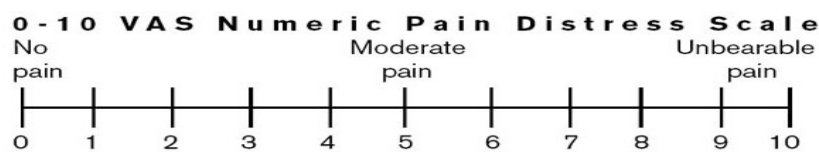

\section{RESULTS}

Total 100 patients were included in this study which were divided into two groups. Mean age of the patients in group A and B was $43.08+11.53$ and $41.70+10.96$ respectively. Out of the 100 patients, 37 were males and rest were females. Males formed $32.0 \%$ ( $n: 16)$ patients in group A while in group $B$, they were $42 \%$ (n: 21 ). (Table-I and II)

Mean pain (VAS 24 hours) in both the groups was $3.16+1.23$ and $5.42+1.01$ respectively. Independent sample t-test was used to compare mean pain (VAS 24 hours) in both the groups which was statistically significant ( $p$-value 0.000 ), as shown in Table-III.

Effect modifier like age was stratified. Among patients with age $18-40$ years, mean pain (VAS 
24 hours) in both the groups was $3.14+1.10$ and $5.54+0.94$ respectively ( $p$-value 0.000 ) whereas among patients with age 41 - 60 years, mean pain (VAS 24 hours) in both the groups was $3.16+1.12$ and $5.42+1.01$ respectively ( $p$-value 0.000). Among male patients, mean pain (VAS 24 hours) in both the groups was $3.19+1.32$ and $5.67+1.06$ respectively ( $p$-value 0.000 ) whereas among female patients, mean pain (VAS 24 hours) in both the groups was $3.15+1.20$ and $5.24+0.95$ respectively ( $p$-value 0.000 ).

There was a single mortality in the Study group B. 6 patients needed ICU care with ventilatory support due to respiratory complications (pleural effusions, ARDS, etc.), 5 were of IV analgesia group and one was of epidural group. The rescue analgesia requirement in both groups was similar and no significant difference in requirement was observed (18\% VS 20\%) (Table-IV).

\section{DISCUSSION}

Effect of EA on pain management in patients with predicted severe Acute Pancreatitis has been well established. ${ }^{5,12}$ Furthermore, the safety of EA has been widely documented in the literature..$^{19,20}$ and its benefit on postoperative morbidity and mortality are well known. ${ }^{23}$ Pancreatitis severity has been studied at a microscopic level and various mechanisms have been proposed to explain in pathogenesis and various reasons for the conversion of mild disease to severe disease have been evaluated.

\begin{tabular}{|l|c|c|c|}
\hline \multicolumn{1}{|c|}{ GROUPS } & $\mathbf{n}$ & Mean Age (years) & Std. Deviation \\
\hline & 100 & 42.39 & 11.21 \\
\hline Group A (Epidural Analgesia-EA) & 50 & 43.08 & 11.53 \\
\hline Group B (Intravenous Analgesia) & 50 & 41.70 & 10.96 \\
\hline
\end{tabular}

Table-I. Age distribution.

\begin{tabular}{|l|c|c|c|}
\hline \multirow{2}{*}{ Male } & \multicolumn{2}{|c|}{ Two Groups } & Total \\
\cline { 2 - 4 } & Epidural Analgesia (EA) & Intravenous Analgesia & 37 \\
\hline \multirow{2}{*}{ Female } & 16 & 21 & $37.0 \%$ \\
\hline Total & $32.0 \%$ & $42.0 \%$ & 63 \\
\hline & 34 & 29 & $63.0 \%$ \\
\hline & $68.0 \%$ & $58.0 \%$ & 100 \\
\hline
\end{tabular}

\begin{tabular}{|l|c|c|c|c|c|}
\hline & Two Groups & n & Mean & Std. Deviation & P-Value \\
\hline Pain score & Epidural Analgesia (EA) & 50 & 3.16 & 1.23 & \multirow{2}{*}{0.000} \\
\hline (VAS as 24 hours) & Intravenous Analgesia & 50 & 5.42 & 1.01 & \\
\hline
\end{tabular}

Table-III. Comparison of Mean Pain (VAS 24 hours) among patients in both the groups.

\begin{tabular}{|c|c|c|c|c|}
\hline & \multicolumn{2}{|c|}{ Two Groups } & \multirow{3}{*}{$\begin{array}{c}\text { Total } \\
19 \\
\end{array}$} \\
\hline & & Epidural Analgesia (EA) & Intravenous Analgesia & \\
\hline \multirow{4}{*}{ Analgesia Requirement } & \multirow{2}{*}{ Yes } & 9 & 10 & \\
\hline & & $18.0 \%$ & $20.0 \%$ & $19.0 \%$ \\
\hline & \multirow{2}{*}{ No } & 41 & 40 & 81 \\
\hline & & $82.0 \%$ & $80.0 \%$ & $81.0 \%$ \\
\hline \multicolumn{2}{|l|}{ Total } & 50 & 50 & 100 \\
\hline
\end{tabular}


The activation of pancreatic enzymes leading to edema and necrosis, vasoconstriction and pancreatic ischemia are few of those. EA increases blood flow and delays metabolic acidosis. These effects have been attributed to a sympathetic nerve blockade that redistributes blood flow to non-perfused regions. ${ }^{4,11}$ EA has a beneficial effect on the severity of AP, suggesting that EA leads to an improvement in pancreatic blood flow with a concomitant decrease in the severity of metabolic acidosis and diminished tissue injury.

Development of pancreatic necrosis is a critical event in AP that determines patient prognosis because it is often accompanied by infection and multiple organ dysfunction syndromes and, thus, is associated with a high mortality. ${ }^{12}$ Therefore, early detection of necrosis is important for the appropriate treatment of predicted severe AP. The literature supports the use of CT scan perfusion studies to measure blood flow and diagnose necrosis in the pancreas. ${ }^{16}$ Pancreatic perfusion showed significant improvement of the parenchymal blood flow within the pancreatic gland in the group treated with EA when compared to the control group on admission and at $72 \mathrm{hrs}$. This observation substantiates the theory that the severity of AP may be related to a vasoconstriction phenomenon, which can be attenuated by EA. ${ }^{1,16}$ It also suggests that the use of EA decreases progression from edematous to severe necrotizing pancreatitis caused by early ischemia of the gland and thus could reduce the severity of the disease. This observation is also reflected in our study with lesser ICU admissions and no mortality in test group.

Our study primarily compared the mean pain scores in patients receiving epidural analgesia and those receiving intravenous analgesia in moderately severe acute pancreatitis. Mean pain (VAS 24 hours) in both the groups was $3.16+1.23$ and $5.42+1.01$ respectively. Comparing it to a study conducted by Sadowski et al, the pain scores were 0.57 vs 2 at 24 hours which are significantly lower in both groups than our study. ${ }^{1}$ The reason could be the differences in expression of pain in different cultures and races and pain being a subjective phenomenon. Nevertheless, the scores were significantly lower in the test groups in both studies.

One of the major limitations of this study was the lack of blinding between the two groups. The major reason for this was the invasive procedure (epidural catheter placement) being done on the test group with its attendant risk of complications and subjecting this procedure to the control group just for the sake of blinding would have raised ethical issues. So, there is a chance of patient as well as observer bias in the pain scores recording. Nevertheless, the lesser rate of respiratory complications, reduced pulse rate etc. show some objective evidence of effective pain relief in the test groups. This study also proposes multicenter trials on this topic so that epidural analgesia becomes a norm in the management of acute severe pancreatitis, rather than a luxury offered to a select few patients.

\section{CONCLUSION}

Epidural analgesia significantly improves pain scores in moderately severe pancreatitis compared to IV analgesia. It also decreases respiratory complications in these patients and decreases progression of disease and thus also improves morbidity and mortality.

Copyright $(23$ Feb, 2021.

\section{REFERENCES}

1. Sadowski SM, Andres A, Morel P, Schiffer E, Frossard $\mathrm{JL}$, Platon $\mathrm{A}$, et al. Epidural anesthesia improves pancreatic perfusion and decreases the severity of acute pancreatitis. World J Gastroenterol. 2015 21; $21: 12448$.

2. Windisch O, Heidegger CP, Giraud R, Morel P, Bühler L. Thoracic epidural analgesia: A new approach for the treatment of acute pancreatitis?. Critical Care. 2016 4; 20:116.

3. Jabaudon M, Belhadj-Tahar N, Rimmelé T, JoannesBoyau O, Bulyez S, Lefrant JY, et al. Thoracic epidural analgesia and mortality in acute pancreatitis: $A$ multicenter propensity analysis. Crit. Care Med. 2018 Mar 1; 46(3):e198-205.

4. Tyagi A, Gupta YR, Das S, Rai G, Gupta A. Effect of segmental thoracic epidural block on pancreatitisinduced organ dysfunction: A preliminary study. Indian J Crit Care Med. 2019 Feb; 23(2):89. 
5. Bulyez S, Pereira B, Caumon E, Imhoff E, Roszyk L, Bernard L, Bühler L, et al. Epidural analgesia in critically ill patients with acute pancreatitis: the multicenter randomised controlled EPIPAN study protocol. BMJ open. 2017 May:e015280.

6. Dong E, Chang JI, Verma D, Butler RK, Villarin CK, Kwok $\mathrm{KK}$, Chen W, Wu BU. Enhanced recovery in mild acute pancreatitis: A randomized controlled trial. Pancreas. 2019 Feb 1; 48(2):176-81.

7. Petrov MS, Yadav D. Global epidemiology and holistic prevention of pancreatitis. Nat Rev Gastroenterol hepatol. 2019 Mar; 16(3):175-84.

8. Kirkegård J, Cronin-Fenton $\mathrm{D}$, Heide-Jørgensen $\mathrm{U}$, Mortensen FV. Acute pancreatitis and pancreatic cancer risk: A nationwide matched-cohort study in Denmark. Gastroenterology. 2018 May 1; 154(6):172936.

9. Peery AF, Crockett SD, Murphy CC, Lund JL, Dellon ES, Williams $\mathrm{JL}$, et al. Burden and cost of gastrointestinal, liver, and pancreatic diseases in the United States: Update 2018. Gastroenterology. 2019 Jan 1; 156(1):25472.

10. Zator Z, Whitcomb DC. Insights into the genetic risk factors for the development of pancreatic disease. Therap Adv Gastroenterol. 2017 Mar; 10(3):323-36.

11. Garber A, Frakes C, Arora Z, Chahal P. Mechanisms and management of acute pancreatitis. Gastroenterol Res Pract. 2018 Mar 15; 2018.

12. Tenner S, Baillie J, DeWitt J, Vege SS. American College of Gastroenterology guideline: Management of acute pancreatitis. The American journal of gastroenterology. 2013 Sep; 108(9):1400.

13. Aden MD, Deng T, Yuting L. Incident of Post ERCP Complications: Meta-analysis and Systematic Review. Archives of Internal Medicine Research. 2020; 3(3):168-77.

14. Cai O, Tan S. From pathogenesis, clinical manifestation, and diagnosis to treatment: An overview on autoimmune pancreatitis. Gastroenterol Res Pract. 2017 Jan 19; 2017.
15. Crockett SD, Wani S, Gardner TB, Falck-Ytter Y, Barkun AN, Crockett $S$, et al. American Gastroenterological Association Institute guideline on initial management of acute pancreatitis. Gastroenterology. 2018 Mar 1; 154(4):1096-101.

16. Mikó A, Vigh É, Mátrai P, Soós A, Garami A, Balaskó $M$, et al. Computed Tomography Severity Index versus other indices in the prediction of severity and mortality in acute pancreatitis: A predictive accuracy meta-analysis. Front. physiol. 2019; 10:1002.

17. Yamashita T, Horibe M, Sanui M, Sasaki M, Sawano $\mathrm{H}$, Goto $\mathrm{T}$, et al. Large volume fluid resuscitation for severe acute pancreatitis is associated with reduced mortality: A multicenter retrospective study. J. Clin. Gastroenterol. 2019 May 1; 53(5):385-91.

18. Almario CV, Ballal ML, Chey WD, Nordstrom C, Khanna $\mathrm{D}$, Spiegel BM. Burden of gastrointestinal symptoms in the United States: Results of a nationally representative survey of over 71,000 Americans. Am J Gastroenterol. 2018 Nov; 113(11):1701.

19. Tenner S, Baillie J, Dewitt J, Vege SS. American College of Gastroenterology guideline: management of acute pancreatitis [published correction appears in Am J Gastroenterol. 2014; 109(2):302]. Am J Gastroenterol. 2013; 108(9):1400-1415.

20. Velissaris D, Karanikolas M, Pantzaris N, Kipourgos G, Bampalis V, Karanikola K, et al. Acute abdominal pain assessment in the emergency department: The experience of a Greek university hospital. J. Clin. Gastroenterol. 2017 Dec; 9(12):987.

21. Ismail OZ, Bhayana V. Lipase or amylase for the diagnosis of acute pancreatitis?. Clin. Biochem. 2017 Dec 1; 50(18):1275-80.

22. Bollen TL, Singh VK, Maurer R, Repas K, Van Es HW, Banks PA. A comparative evaluation of radiologic and clinical scoring systems in the early prediction of severity in acute pancreatitis. The American journal of gastroenterology. 2012 Apr; 107(4):612.

23. Xiao B, Xu HB, Jiang ZQ, Zhang J, Zhang XM. Current concepts for the diagnosis of acute pancreatitis by multiparametric magnetic resonance imaging. Quant Imaging Med Surg. 2019 Dec; 9(12):1973. 


\section{AUTHORSHIP AND CONTRIBUTION DECLARATION}

\begin{tabular}{|c|c|c|c|}
\hline Sr. \# & Author(s) Full Name & Contribution to the paper & Author(s) Signature \\
\hline 1 & Ghulam Khadija & Concept design. & \\
\hline 2 & Waqas Hasan Syed & $\begin{array}{l}\text { Drafting of article }+ \text { Proof } \\
\text { reading. }\end{array}$ & \\
\hline 3 & Sana Ullah & Collection of data. & \\
\hline 4 & Munazzah Aziz & Collection of data, Literature. & \\
\hline 5 & Sumaira Nawaz & Literature search. & \\
\hline 6 & Abeera Zareen & $\begin{array}{l}\text { Overriding of international } \\
\text { design. }\end{array}$ & \\
\hline 7 & Mehwish Changeez & Literature design. & \\
\hline 8 & Sara Malik & Statistical support. & \\
\hline 9 & Maham Tariq & Statistical support. & \\
\hline 10 & Jahangir Sarwar Khan & Proof reading. & \\
\hline
\end{tabular}

